\title{
Maßgeschneiderte Konzepte in der Hepatologie \\ nate
}

\begin{abstract}
In der Hepatologie vollzieht sich derzeit ein Paradigmenwechsel: „Es geht zunehmend darum, die verfügbaren Behandlungsoptionen weiterzuentwickeln und für die Patienten quasi maßgeschneiderte Therapiekonzepte zu erarbeiten“, erklärte Professor Dr. Michael Manns, Hannover, dort bei einem Falk-Workshop im Vorfeld der Jahrestagung der German Association for the Study of the Liver (GASL).
\end{abstract}

Zwar steht die „Targeted Therapy“ bei Lebererkrankungen noch am Anfang, der Weg in eine maßgeschneiderte Therapie ist jedoch bei verschiedenen Krankheitsbildern vorgezeichnet. Neben den chronischen viralen Hepatitiden, bei denen in den vergangenen Jahren erhebliche therapeutische Fortschritte zu verzeichnen waren und die Behandlung zunehmend individualisiert erfolgt, nannte Manns als Beispiel die cholestatischen Lebererkrankun- gen sowie die Autoimmunhepatitis. So wird zum Beispiel daran gearbeitet, die klinische Wirksamkeit der bei der primär biliären Zirrhose (PBC) routinemäßig eingesetzten Ursodeoxycholsäure (UDC) zu verbessern. Ziel dabei ist es, durch die Weiterentwicklung der Gallensäure auch eine effektive medikamentöse Therapieoption bei der primär sklerosierenden Cholangitis (PSC) etablieren zu können. Hoffnungen setzen die Hepatologen vor allem auf die norUDC, ein Homolog der UDC, die sich von dieser durch eine modifizierte Seitenkette des Moleküls unterscheidet. Dies bedingt den ersten Befunden zufolge eine höhere Anreicherung in der Gallenflüssigkeit. Dadurch bildet sich laut Prof. Dr. Michael Trauner, Wien eine Art „Bicarbonat-Schutzschirm“ aus, der die Gallenwege offenbar besser vor Schädigungen bewahren kann als herkömmliche Therapeutika.

\section{Bessere Verträglichkeit \\ Nicht immer geht es bei therapeutischen Fortschritten darum, gezielt die Effektivität der Behandlung zu steigern. Das Ziel kann nach Prof. Dr. Michael Manns, Hannover, auch darin bestehen, die Behandlung bei gleicher Effektivität verträglicher zu machen, wie das Beispiel der Autoimmunhepatitis demonstriert.}

Das Krankheitsbild erfordert eine langfristige Behandlung, wobei die Standardtherapie in der Gabe von Steroiden besteht. Bislang wurde vorwiegend mit Prednison behandelt, die Patienten aber haben oft mit erheblichen Nebenwirkungen zu kämpfen wie der Entwicklung einer Akne, eines
Mondgesichtes und/oder eines Stiernackens. Deutlich minimieren lässt sich das Risiko solcher Nebenwirkungen, wenn statt Prednison Budesonid gegeben wird.

Das Steroid wird in der Asthmatherapie routinemäßig eingesetzt. Es entfaltet auch bei Lebererkrankungen analog der

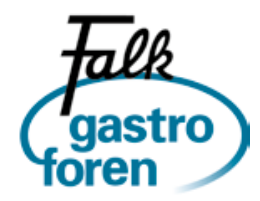

Besuchen Sie das nächste Falk Gastro Forum „Therapeutische Herausforderungen 2013 in Gastroenterologie und Hepatologie" am Samstag, den 4. Mai 2013 in München; Infos: www.drfalkpharma.de/veranstaltungen

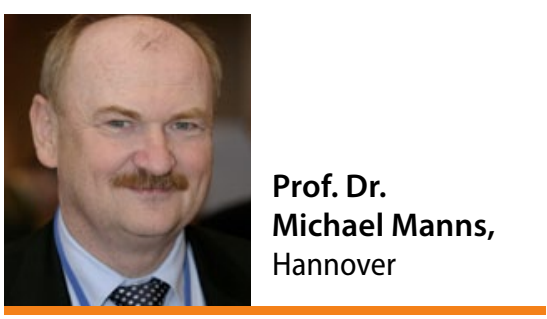

Inhalationstherapie beim Asthma quasi eine lokale Wirksamkeit. Denn Budesonid unterliegt einem hohen First Pass-Effekt, wird also praktisch sofort metabolisiert und es resultiert damit eine nur sehr geringe systemische Bioverfügbarkeit. „Wir nutzen somit gezielt die besondere Pharmakokinetik des Wirkstoffs, um die Behandlung für die Patienten verträglicher zu machen“, so Manns. Studien belegen, dass steroidtypische Begleitreaktionen der Therapie signifikant seltener sind als unter Prednison. Kontraindiziert ist der Wirkstoff allerdings bei Patienten mit manifester Leberzirrhose.

(CV)

Falk Workshop "Targeted Therapies in Hepatology", 24.-25. Januar 2013 in Hannover im Vorfeld der GASL-Jahrestagung (German Association for the Study of the Liver) 